\title{
Credit where credit is due
}

\author{
A proposed author ID system is gaining widespread support, and could help lay the foundation for an \\ academic-reward system less heavily tied to publications and citations.
}

( $\mathrm{n}$ his classic book Management Teams, UK psychologist Meredith Belbin used extensive empirical evidence to argue that effective teams require members who can cover nine key roles. These roles range from the creative 'plants' who generate novel ideas, to the disciplined 'implementers' who turn plans into action and the big-picture 'coordinators' who keep everyone working together.

Much the same range of roles is critical for science. Unfortunately, the academic system tends to reward only some of those activities - notably those that have easily measured outcomes, such as the publication and citation numbers so heavily weighted by promotion and tenure committees.

On page 843, Nature profiles a research group composed largely of what Belbin would call 'completer finishers' - perfectionists who are driven to fix all the flaws, fill all the gaps and get the job finished correctly. This particular group is trying to complete the reference human genome sequence, which is still full of errors nearly a decade after the first draft was announced in 2000. It is essential work: modern sequencing techniques still use the reference to anchor new data even as they grind out genomes at a fraction of the cost of the original. But it is also work that offers few academic rewards beyond the satisfaction of a job well done - it is unlikely to result in a highprofile publication.

Such unsung contributions to science may soon be easier to evaluate and quantify through an author ID system proposed earlier this month and backed by 23 organizations, including Thomson Reuters, Nature Publishing Group, Elsevier, ProQuest, Springer, CrossRef, the British Library and the Wellcome Trust. The Open Researcher and Contributor ID (ORCID) would be an alphanumeric string that uniquely identifies an individual scientist in much the same way that a Digital Object Identifier uniquely identifies a paper, book or other scholarly publication (more details and the complete list of participants will soon be available at www.orcid.org). The system would distinguish between the world's multitudinous Dr Smiths and Professor Wangs, but would not be affected by name changes, cultural differences in name order, inconsistent first-name abbreviations or the use of different alphabets. It would be attached to researchers' journal publications, and could also be assigned to data sets they helped to generate, comments on their colleagues' blog posts or unpublished draft papers, edits of Wikipedia entries and much else besides.

This kind of 'microattribution' could ultimately make it possible for each researcher to have a constantly updated 'digital curriculum vitae' providing a picture of his or her contributions to science going far beyond the simple publication list.

ORCID is hardly the first proposal for an author ID system. A number of publishers have been exploring the idea, and the International Organization for Standardization in Geneva is developing an international standard name identifier to track contributors to media content such as books, television programmes and newspaper articles. But most of those developers have already joined or are working closely with the ORCID group. Moreover, the intention is to make ORCID freely available for anyone to use, and interoperable with existing ID systems.

"The ID system could make it possible for each researcher to have a constantly updated 'digital curriculum vitae'."

The next step is for the ORCID group to turn the concept into a working system. That is scheduled to happen over the next six months, with the software being based on Thomson Reuters' existing ResearcherID system. In parallel, the group will be setting up an independent organization to run the system and assign ORCIDs to individual researchers.

There will be many challenges along the way — not least of which is establishing rigorous protocols for validating and authenticating ORCID assignments. No one wants to see the system abused by individuals seeking to pad their academic credentials.

But perhaps the largest challenge will be cultural. Whether ORCID or some other author ID system becomes the accepted standard, the new metrics made possible will need to be taken seriously by everyone involved in the academic-reward system - funding agencies, university administrations, and promotion and tenure committees. Every role in science should be recognized and rewarded, not just those that produce high-profile publications.

\section{Mind the gap}

\section{It will take time to assess the value of fresh approaches to science and technology studies.}

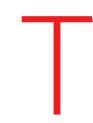

he relationship between the social sciences and the natural sciences has historically been fraught. 'Hard' scientists have often treated the social sciences with disdain. For example, some of them fought, successfully at first, to exclude the social sciences from the remit of the US National Science Foundation. And those social scientists who studied science itself, under the remit of science and technology studies, often returned the favour, seeming on occasion to be devoting themselves myopically to demonstrating that the scientific emperor had few, if any, clothes.

There remains something of a dialogue of the deaf between these two wings of the academy, separated as they are by language, custom and methodology. But barriers are coming down. Senior scientists and administrators, especially those in socially contentious areas such as climate change and reproductive technologies, realize that 